\title{
Newsfronts
}

\section{Diabetic Mice Cured through Gene Therapy}

Researchers have used gene therapy to cure diabetes in mice by inducing liver cells to secrete several key hormones, including insulin. This research may open the door to promising diabetes therapies for humans in the future.

Diabetics fail to produce or properly use insulin, a hormone that allows glucose in the bloodstream to enter cells; consequently, they suffer from hyperglycemia and require daily insulin shots. Gene therapy would offer a promising alternative to current therapies because, in theory, it coaxes the body to produce its own insulin.

Lawrence Chan, a researcher at the Baylor College of Medicine (Houston, TX), and his colleagues used an adenovirus vector to introduce two genes - Neurod and $B t c$ - into the livers of mice that had been treated with streptozocin (STZ) to induce diabetes (Nature Medicine, May 2003). Neurod is a transcription factor required for the proper development of pancreatic islets, the group of cells that produce hormones involved in glucose metabolism. Btc is a hormone that stimulates the growth of $\beta$-cells, the islet cells that specifically secrete insulin. The treatment led to a complete reversal of the mice's diabetic condition. Neurod-Btc-treated mice developed large islet clusters in their livers, producing the hormones insulin, glucagon, pancreatic polypeptide, and somatostatin. Within three weeks, STZ mice treated with NeurodBtc showed normal blood-serum levels; three months later they still showed normal insulin and glucose levels.

"The most significant aspect of our work is that we have proof of principle that you can turn cells in the liver into islets," Chan tells Lab Animal. "Prior to our work, many people would not have believed that this could possibly happen. This discovery will stimulate a lot of research in the area." Indeed, the results of the study suggest a promising gene therapy regimen for the treatment of insulin-dependent diabetes in humans, although the technique will require considerable further development. Chan plans to repeat the experiments in

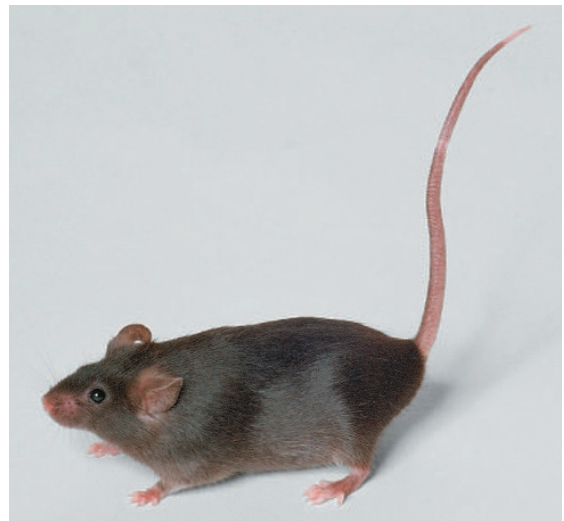

larger animals, and to continue researching the safest vectors for gene delivery. In addition, he would like to explore the possibility of replicating the experiment in vitro. $\mathrm{He}$ tells Lab Animal, "If we can eventually do this in people, the islets that we generate in the test-tube can be used for transplantation back to the same patient, thus avoiding [the need for] immunosupression and the [problems associated with the limited] availability of donors."

\section{-Derek Lee}

\section{Bright, Sexy, and Immunocompetent}

The most attractive males are also the healthiest, according to the results of a new study in finches.

Although scientists have long observed that females in many animal species prefer to mate with the most elaborately ornamental males, the biological and evolutionary underpinnings behind this behavior are still not fully known. Now, a group led by Jonathan D. Blount of the University of Glasgow (Glasgow, UK) has found compelling evidence that supports a leading hypothesis that brilliant ornamentation is indicative of superior health and immunocompetence.

Carotenoids are red and yellow pigments associated with the brightly colored ornamentation in several animal species, such as flamingos and anemone fish; they are also known antioxidants and immunostimulants. Since animals cannot synthesize their own carotenoids, they must obtain them exclusively through their diets. Working with full-sibling pairs of male zebra finches (Taeniopygia guttata), Blount's team gave drinking water fortified with the carotenoids lutein and zeaxanthin to experimental group finches over a tenweek period; their brothers in the control group were given normal, distilled water (Science, 4 April 2003).

While both groups started the study with the same color ornamentation, the finches in the experimental group soon developed significantly redder bills. Female finches were far more attracted to these finches than to their control group brothers, as measured by the relative proportion of time females spent perching next to individual males. Compared with the controls, the experimental group had a significantly higher concentration of circulating carotenoids and showed stronger reactions when injected with phytohemagglutinin, a lectin used to test the strength of immune responses.

These results suggest that immunocompetence is revealed by carotenoid-dependent ornamentation in certain species. The possible benefits for the female in choosing a highly ornamental male include a decreased risk of infection as well as an increased probability of passing onto her offspring genes that confer enhanced resistance to disease, parasites, and oxidative stress. "The question of why the showiest males are preferred by females has been of interest to biologists for over 130 years, since Darwin laid the foundations of the study of evolutionary biology," Blount tells Lab Animal. "Since carotenoid-dependent sexual traits are common among bird species, and also among some other animal groups, we have to consider the possibility that sexual signaling of immunocompetence could be a widespread phenomenon in nature."

Blount's group plans to study additional factors involved in carotenoid acquisition and their subsequent use in sexual displays, such as energy use in carotenoid metabolism and foraging efficiency. According to Blount, "The relative importance of these factors in driving the evolution of carotenoid-dependent sexual displays remains to be seen." 